\title{
DNA Damage-Induced NF- $\kappa$ B Activation in Human Glioblastoma Cells Promotes miR-181b Expression and Cell Proliferation
}

\author{
Rui-Xue Xua Rong-Yao Liua Chun-Ming Wua Yong-Shun Zhao ${ }^{a}$ Yang Lia \\ Yi-Qun Yao ${ }^{a}$ Ying-Hui Xu \\ ${ }^{a}$ Department of Neurosurgery, the First Affiliated Hospital of Dalian Medical University, Dalian, China
}

\section{Key Words}

Glioblastoma • Radioresistance • DNA damage • NF-kB signaling • miR-181b

\begin{abstract}
Background: Glioblastoma (GBM) is the most common and most aggressive form of brain cancer. After surgery, radiotherapy is the mainstay of treatment for GBM patients. Unfortunately, the vast majority of GBM patients fail responding to radiotherapy because GBM cells remain highly resistant to radiation. Radiotherapy-induced DNA damage response may correlate with therapeutic resistance. Methods: Ionizing radiation (IR) was used to induce DNA damage. Cell proliferation and migration were detected by wound-healing, MTT and apoptosis assays. Dual-luciferase assays and Western blot analysis were performed to evaluate NF-KB activation and validate microRNA targets. Real-time PCR was used to study mRNA and microRNA levels. Results: IR-induced DNA damage activated NF-KB in GBM cells which promoted expression of IL-6, IL-8 and BCl-xL, thereby contributing to cell survival and invasion. Knockdown SENP2 expression enhanced NF-KB essential modulator (NEMO) SUMOylation and NF-KB activity following IR exposure. miR-181b targets SENP2 and positively regulated NF-KB activity. Conclusion: NF-KB activation by DNA damage in GBM cells confers resistance to radiationinduced death.

Copyright (C) 2015 S. Karger AG, Basel
\end{abstract}

\section{Introduction}

Glioblastoma (GBM) is the most common malignant primary central nervous system tumor in adults. Despite significant progress has been made in radiation, chemotherapy, and targeted biologic therapy, outcomes remain poor $[1,2]$. A reason why it is very difficult to treat GBM is therapeutic resistance [3, 4]. Nevertheless, the exact mechanisms of therapeutic resistance remain poorly understood.

Yinghui Xu

KARGER 125
Department of Neurosurgery, The First Affiliated Hospital of Dalian Medical University, No. 222 Zhong Shan Road, Dalian 116011 (P.R. China)

Tel. +86-411-83622844, Fax +86-411-83622844, E-Mail YinghuiXu2013@126.com 
The transcription factor NF- $\kappa$ B plays a crucial role in the regulation of immune and inflammatory responses through regulating the expression of inflammatory cytokines and chemokines and, in turn, induced by them [5-7]. There is accumulating evidence to suggest that deregulated NF- $\kappa B$ activity is a hallmark of most human cancers [8, 9]. Moreover, the antiapoptotic function of NF- $\mathrm{\kappa B}$ is believed to promote resistance to common chemotherapeutic drugs and ionizing radiation (IR) in a number of cancer types, including breast cancer, hepatocellular carcinoma and gastric cancer [6,10-12]. DNA damage is therapeutically induced for cancer treatment with the aim to promote apoptotic death. Unfortunately, DNA damage also induced NF- $\mathrm{\kappa B}$ activation in several human cancers which has been seen to protect damaged cells from apoptotic death, thereby contributing to therapeutic resistance $[6,13,14]$. Following DNA damage, ataxia telangiectasia mutated (ATM) was activated and post-translational modifications of NEMO, including SUMOylation, phosphorylation and ubiquitination were promoted. These nuclear events cooperate to activate cytoplasmic TGF$\beta$-activated kinase 1 (TAK1) and IKK to promote NF- $\kappa B$ activation through multiple signal transduction mechanisms depending on the severity of genotoxic stress and cell type [1416]. Negative regulation of genotoxic NF- $\kappa B$ signaling could provide a method of enhancing the effectiveness of cancer therapy. SUMOylation of NEMO is a critical signaling event to mediate NF- $\kappa \mathrm{B}$ activation in the DNA damage signaling. SENP2 has been identified as the primary SUMO protease that deSUMOylates NEMO in vivo [17]. Overexpression of SENP2 leads to NEMO deSUMOylation and decreased NF- $\kappa B$ activation upon DNA damage. More interestingly, SENP2 expression can be induced by NF- $\kappa B$ in response to DNA damage by an unknown mechanism [18]. Thus, NF- $\kappa B$-dependent SENP2 induction may be a generalized self-limiting response to DNA damage.

In addition to regulating protein-coding genes, accumulating evidence indicates that NF$\kappa \mathrm{B}$ also regulates microRNA expression $[19,20]$. MicroRNAs (miRNAs) are a class of highly conversed short non-coding RNA molecules (containing about 22 nucleotides) that regulate diverse cellular processes by binding to the 3'-untranslated region (3'-UTR) of target mRNAs resulting in direct cleavage of the targeted mRNAs or translation inhibition. Some miRNAs may function as oncogenes or tumor suppressors and expression of miRNA is frequently dysregulated in human malignancies [21, 22]. miR-181b was shown to be highly expressed in colorectal, head and neck, and bladder cancer, but be down-regulated in glioblastoma, indicating that the function of miR-181b may depend on the type of tumor and cellular context [22]. Iliopoulos et al present work that transient expression of miR-181b is sufficient to initiate an epigenetic switch from inflammation to cancer by direct targeting of CYLD, an inhibitor of NF- $\kappa B$, leading to increased NF- $\kappa B$ activity acquired to maintain the transformed state [23]. The biological significance of miR-181b in regulation of DNA damage-induced NF$\kappa B$ activity and cellular sensitivity to genotoxic treatment remain obscure.

In the present study, we show that IR exposure activates NF- $\kappa B$ significantly in human GBM cells and promotes cell proliferation and miR-181b expression. Up-regulation of miR$181 \mathrm{~b}$ by IR in turn enhances NF- $\kappa B$ activity by directly targeting SENP2. Thus, constitutive NF- $\kappa B$ activation in GBM cells may confer resistance to IR-induced cell death.

\section{Materials and Methods}

Cell lines, plasmids, antibodies and reagents

T98G cells (human glioblastoma multiforme cell line) and U87MG cells (human glioblastomaastrocytoma, epithelial-like cell line) were cultured in Dulbecco's modified Eagle's medium (DMEM) supplemented with $10 \%$ heat-inactivated fetal bovine serum (Gibco, Paisley, United Kingdom). The reporter plasmid pNF-кB-Luc was obtained from Clontech and pRL-TK and pGL3-control vectors were obtained from Promega. Wild-type or mutated miR-181b putative targets on SENP2 3'-UTR were cloned into the Xba I site of pRL-TK vector. Briefly, the 3'- UTR of human SENP2 gene (-151- -375) was obtained by PCR using the following primers: 5'- CCGTCTAGACTAGTCCTGACTTGGGGTGCAGAG-3' (forward) and 5'-CCGTCTAGAATGTTTG CAAACGAGTGAGTG -3' (reverse). To introduce mutations into the seed sequences of the predicted miR-181b target sites within the SENP2 3'-UTR, recombinant PCR was performed using

\section{KARGER}


Xu et al.: Radiation Induced NF-кB Functions as a Tumor Promoter

the mentioned primers and the following primers: 5'- CCCTATGCCGTTGGGAAATGCAGG -3' (forward) and 5'- CCTGCATTTCCCAACGGCATAGGG -3' (reverse). SENP2 and NEMO were cloned into pcDNA3.0-Flag vector and SUMO was cloned into pXJ40-HA vector. Bay11-7082, broad-spectrum caspase inhibitor Z-VAD-FMK (sc-3067), mouse anti-HA (sc-7392), anti-p-IкB $\alpha$ (sc-8404), anti-Bcl-2 (C-2, sc-7382), anti-Bcl-xL(H-5, sc8392), anti-IKK $\alpha / \beta$ (sc-7607) and anti- $\beta$-actin (C4; sc-47778) antibodies, and rabbit anti-SENP2 (H-300, sc-67075), anti-TAK1 (M-579, sc-7162) and anti-IкB $\alpha$ (C-21; sc-371) antibodies were purchased from Santa Cruz Biotechnology (CA, USA). The mouse anti-Flag (M2; F3165) antibody was purchased from

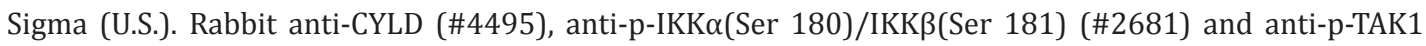
(Ser412) (\#9339) antibodies were purchased from Cell Signaling Technology (USA). miR-181b, miR-181b inhibitor (as-miR-181b), miR negative control (miR-NC), and siRNA oligonucleotides targeting SENP2 were chemically synthesized in RIBOBIO (Guangzhou, China). The sequences for SENP2 siRNAs were as follows: si-SENP2-1: 5'- GUACCGAAAGUUAUUGGAA dTdT-3'; si-SENP2-2: 5'- GGAGCCUGACCUAUCAGAA dTdT-3'; siSENP2-3: 5'- GGACAAACCUAU CACAUUUdTdT-3'.

\section{Cell transfection and radiation treatment}

The plasmids, microRNAs or siRNAs were transfected into T98G or U87MG cells using Lipofectamine ${ }^{\mathrm{TM}}$ 2000 reagent according to the manufacturer's instructions (Invitrogen). To increase the knockdown efficiencies, the cells were transfected with control siRNAs or siRNAs against SENP2 for the second time after $24 \mathrm{~h}$. The transfected cells were irradiated (GE 3000) at a dose rate of $2.0 \mathrm{~Gy} / \mathrm{min}$.

\section{Immunofluorescence assay}

For immunofluorescence, T98G or U87MG cells were grown on cover slips and irradiated with 5 Gy in the presence or absence of general caspase inhibitor z-VAD-FMK $(20 \mu \mathrm{M})$. At 40 min post-treatment, the cells were washed with PBS, fixed in 4\% paraformaldehyde for $20 \mathrm{~min}$ at room temperature and permeabilized with $0.2 \%$ Triton X-100 for $3 \mathrm{~min}$. After blocking in 5\% BSA for $30 \mathrm{~min}$, the cells were incubated with antip-H2A.X ( $\gamma$-H2A.X, \#9718S) monoclonal antibody at $37^{\circ} \mathrm{C}$ for 40 min followed by washing with PBS and incubation with TRITC-conjugated or FITC-conjugated anti-rabbit IgG antibody for $40 \mathrm{~min}$ at $37^{\circ} \mathrm{C}$. The cells were then observed under an Olympus confocal microscope. The fluorescence intensities of $\gamma$-H2A.X were measured with Olympus Confocal Software (FV10-ASW 2.0).

\section{Wound-healing assay}

Confluent cells were scratched with a sterile pipette tip and washed with PBS. Photographs were taken after incubation for 0 and $20 \mathrm{~h}$. Relative cell migration distance was determined by measuring the wound width on the monolayer under a microscope (Leica). The results are presented as the percentage of wound as follows: percentage of wound $=$ (wound width at the time of measurement/initial wound width $) \times 100$.

\section{Cell proliferation assay (MTT)}

T98G cells were seeded in a 96-well plate and exposed to IR (5 Gy) or left untreated along with incubation with Bay-11(10 $\mu \mathrm{M})$ for $4 \mathrm{~h}$. At 12, 24 and $48 \mathrm{~h}$ post-treatment, cell viability was measured using the methylthiazolete- trazolium (MTT) method.

\section{Apoptosis assay}

T98G cells were seeded in a 6-well plate and exposed to IR (5 Gy) or left untreated along with incubation with Bay-11for $4 \mathrm{~h}$. After $20 \mathrm{~h}$, the cells were washed, resuspended in the staining buffer, and examined with Annexin V FITC apoptosis detection kit (BD) according to the manufacturer's instructions. Stained cells were analyzed by fluorescence-activated cell sorting (BD FACScalibur ${ }^{\mathrm{TM}}$ ).

\section{Dual-luciferase reporter assay}

To detect NF-kB activity, T98G or U87MG cells were co-transfected with pNF-kB-Luc reporter plasmid and the internal control pRL-TK. At 28 hours post-transfection, the cells were irradiated with 0,5 and 10 Gy for another $8 \mathrm{~h}$. The cells were harvested, and luciferase activity was measured using the dual-luciferase reporter assay system (Promega, USA) according to the manufacturer's recommendations.

To determine whether miR-181b plays a direct role in repression of luciferase expression from the pRL-TK vector containing SENP2 3'-UTR sequence, T98G or U87MG cells were transfected with miR-181b 
Xu et al.: Radiation Induced NF-KB Functions as a Tumor Promoter

or its negative control along with pRL-TK vector containing wild type or mutated SENP2 3'-UTR sequence, pGL3-control vector as an internal control. At 36 hours post transfection, the cells were harvested for luciferase assay as mentioned above.

\section{Western blot analysis}

Cell lysates were prepared using cell lysis buffer (Cell Signaling Technology). The extracted proteins were separated using SDS-PAGE and transferred to a PVDF membrane. After blocking, the membrane was incubated with the primary antibody overnight at $4^{\circ} \mathrm{C}$. After washing three times with TBS-T, the membrane was incubated with a horseradish peroxidase-conjugated secondary antibody for 2 hours at room temperature. Bands were detected using enhanced chemiluminescence (Applygen, Beijing, China).

\section{Immunoprecipitation (IP) assays}

T98G cells were harvested and lysed in lysis buffer (1\% Triton X-100, $150 \mathrm{mM} \mathrm{NaCl}, 20 \mathrm{mM}$ Hepes, pH 7.5, 10\% glycerol, $1 \mathrm{mM}$ EDTA and protease inhibitors). Cell lysates were centrifuged and the supernatant was incubated with the primary antibody at $4^{\circ} \mathrm{C}$ for 2 hours. Protein G agarose beads (Sigma, U.S.) were then added, and the mixture was incubated at $4^{\circ} \mathrm{C}$ overnight. The beads were washed three times with lysis buffer, boiled in $2 \times$ SDS loading buffer for 5 minutes, and then analyzed by Western blotting.

\section{Quantitative real-time PCR ( $q R T$-PCR)}

For mRNA analysis, total RNA was isolated from cells using TRIzol and then converted to first-strand cDNA using M-MLV reverse transcriptase (Promega, U.S.). Real-time PCR was conducted using SYBR premix Ex Taq II (Takara Co., LTD). The cycle conditions included an initial denaturation step at $95^{\circ} \mathrm{C}$ for 30 seconds followed by 40 cycles of amplification for 3 seconds at $95^{\circ} \mathrm{C}$ and 1 minute at $60^{\circ} \mathrm{C}$. The housekeeping gene GAPDH was used as an internal control. The sequences of gene specific primers used for qRT-PCR were as follows [17, 24, 25]: IL-6, 5'- TACCCCCAGGAGAAGATTCC-3' (forward), 5'- TTTTCTGCCAGTGCCTCTTT-3' (reverse); IL-8, 5'-CCTAACCATGAACGGCAAGC-3' (forward), 5'-ACCAGC GTCCTACCTTGCG-3' (reverse); Bcl-2, 5'-TGGGATGCCTTTGTGGAACTAT-3' (forward), 5'-AGAGACAGCCAGGAGAAATCAAAC-3' (reverse); Bcl-xL, 5'-CTGGGACACTTTTGTGGATCTCT-3'(forward), 5'-GAAGCGCTCCTGGCCTTT- 3' (reverse); SENP2, 5'-TGAAGAGGCGGCGCTCAGACAG-3' (forward), 5'-TCTCTTCAGAAATCATCTCTGT-3' (reverse), GAPDH, 5'-CCCCAATGTCTCTGTTGTTGAC-3'(forward), 5'-CAGCCTTCACTACCCTCTTG AT-3' (reverse).

For the analysis of miR-181b expression, total RNA was poly(A)-tailed using miRcute miRNA firststrand cDNA synthesis kit and real-time PCR was conducted using miRcute miRNA qPCR Detection (SYBR Green) (TianGen Biotech Co., LTD, China) according to the manufacturer's instructions. U6 was used as an internal control. The cycle conditions included an initial denaturation step at $94^{\circ} \mathrm{C}$ for 120 seconds followed by 45 cycles of amplification for 20 seconds at $94^{\circ} \mathrm{C}$ and 34 seconds at $60^{\circ} \mathrm{C}$. The $2^{-\Delta \Delta \mathrm{CT}}$ equation was used to calculate the relative levels of miRNA expression [26]. The sequences of gene specific primers used for qRTPCR were as follows: miR-181b, 5' -GGCCGAACATTCATTGCTGTCGGTGGGT-3'; U6, 5' -CGCAAGGATGACACG CAAATTC- 3'.

\section{Statistical analysis}

The results are presented as the mean \pm S.D. of three independent experiments. Statistical comparisons were performed using SPSS 11.5. Parametrical data were compared using Student's $t$ test. One-way ANOVA analysis was used to determine the difference between independent groups. $P<0.05$ was denoted as statistically significant.

\section{Results}

Ionizing radiation (IR) activates $N F-\kappa B$ in human GBM cells

Human GBM cell lines T98G and U87MG were exposed to IR (5 Gy) to induce DNA damage. Immunofluorescence analysis of phosphorylation of $\mathrm{H} 2 \mathrm{AX}(\gamma-\mathrm{H} 2 \mathrm{AX})$, a biomarker of DNA damage induced by IR [27], suggested that IR exposure caused DNA damage in GBM cells (Fig.1A). To further confirm the increased $\gamma$-H2AX is due to an increase in IR-induced DNA damage rather than caspase cleavage-mediated DNA fragmentation which also induces 
A
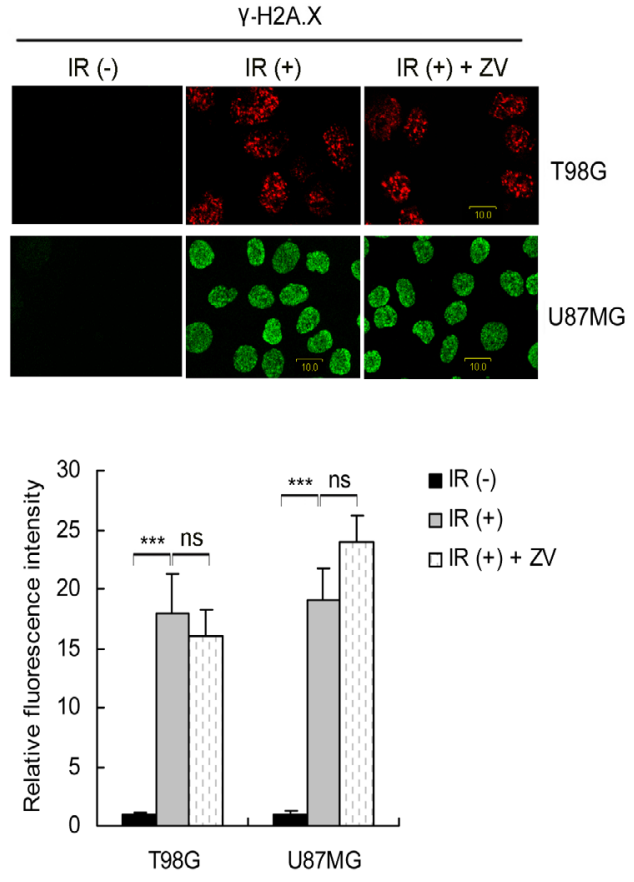

B
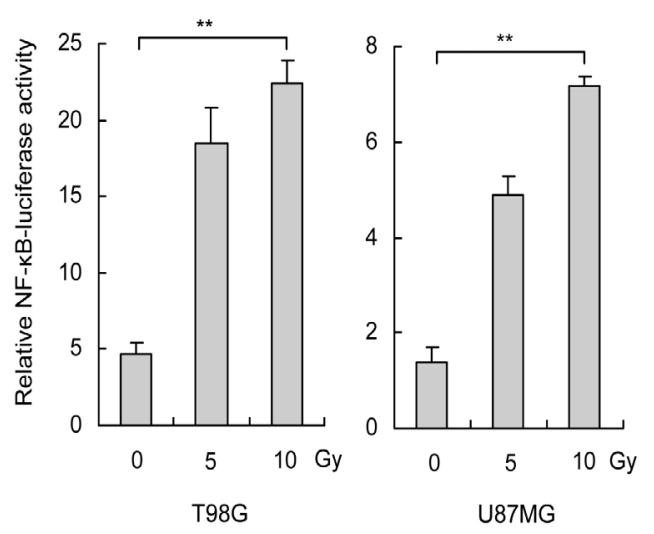

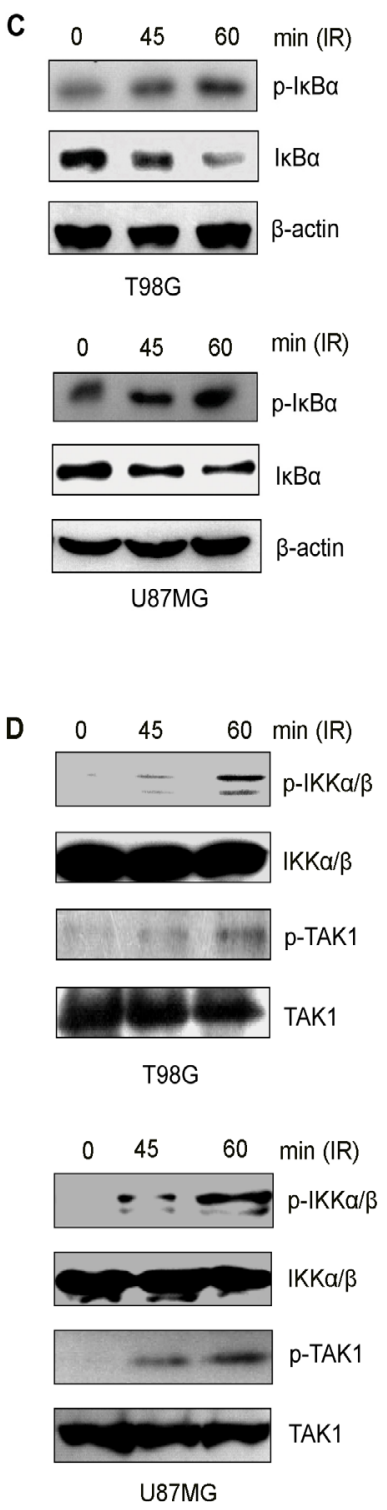

Fig. 1. Ionizing radiation (IR) induces NF- $\mathrm{B}$ activation in human glioblastoma T98G and U87MG cells. (A) T98G or U87MG cells were irradiated (5Gy) in the presence or absence of general caspase inhibitor z-VADFMK $(20 \mu \mathrm{M})$ or left untreated. At 40 min post-treatment the cells were immunostained for $\gamma$-H2A.X. Relative fluorescence intensities of $\gamma$-H2A.X were measured with Olympus Confocal Software (FV10-ASW 2.0). (B) T98G or U87MG cells were transfected with pNF-кB-Luc and pRL-TK for $28 \mathrm{~h}$ followed by IR exposure. At $8 \mathrm{~h}$ post-IR, the cells were harvested, and a luciferase assay was performed. (C-D) T98G or U87MG cells were treated with IR ( $5 \mathrm{~Gy}$ ). Whole-cell lysates were prepared the indicated time points and analyzed by

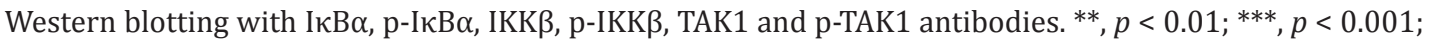
ns, no significant difference.

phosphorylation of H2AX, T98G or U87MG cells were exposed to IR in the presence or absence of general caspase inhibitor z-VAD-FMK $(20 \mu \mathrm{M})$. As shown in Fig. 1A, z-VAD-FMK treatment had no significant effect on the levels of $\gamma$-H2AX in IR-treated cells, suggesting that the increased phosphorylation of H2AX was induced by IR.

We then performed dual-luciferase reporter assays to explore whether IR-induced DNA damage promotes NF- $\mathrm{BB}$ activation in GBM cells. Luciferase assays showed that DNA 


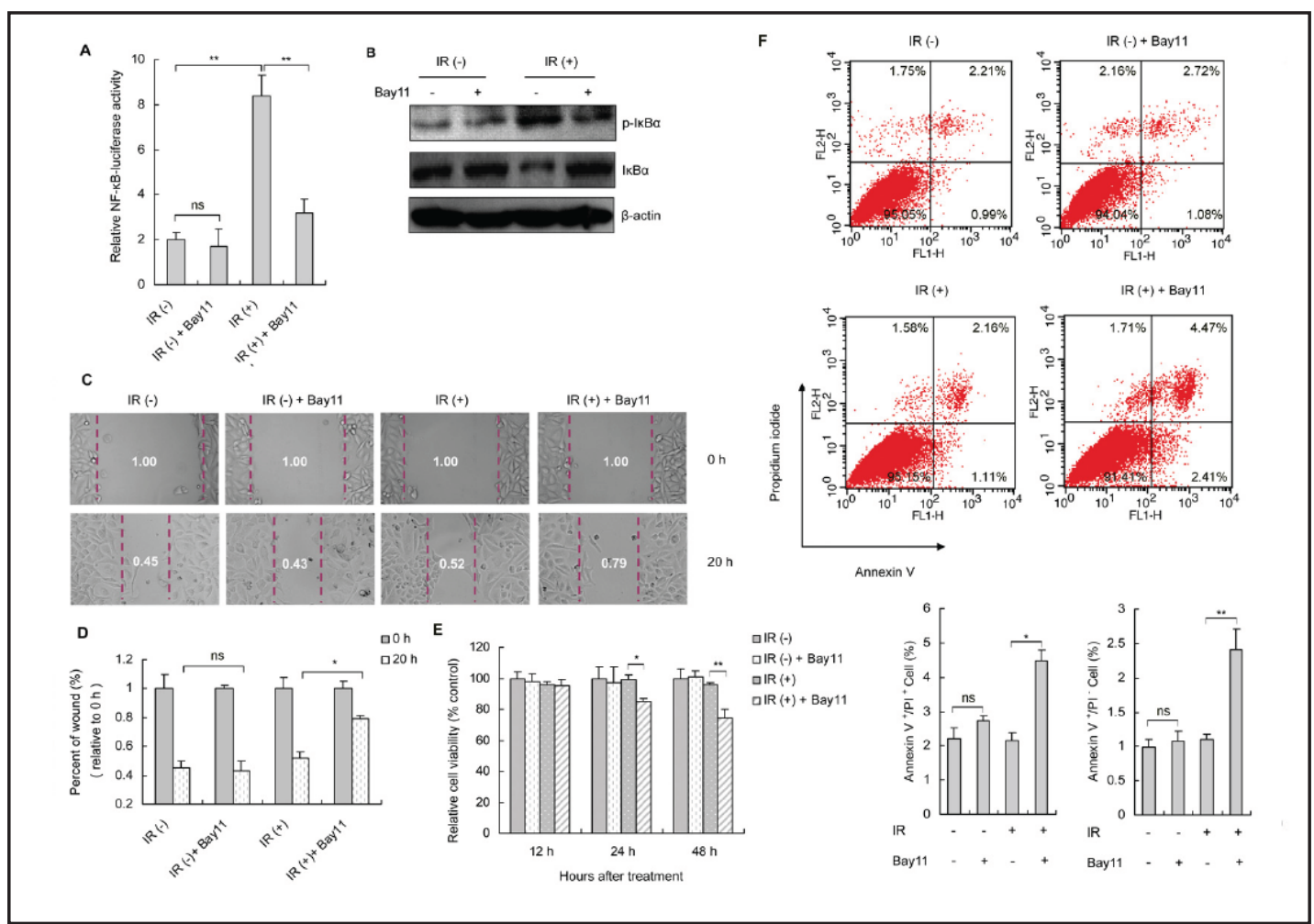

Fig. 2. Inhibition of NF- $\kappa B$ activity increases radiation sensitivity of T98G cells.(A) T98G cells were transfected with pNF-кB-Luc and pRL-TK for $28 \mathrm{~h}$ followed by IR exposure (5 Gy) or left untreated along with incubation with Bay-11. At $8 \mathrm{~h}$ post-IR, the cells were harvested, and a luciferase assay was performed. (B) T98G cells were incubated with Bay-11 for $3 \mathrm{~h}$ followed by IR exposure (5 Gy) for another $1 \mathrm{~h}$. Whole-cell ly-

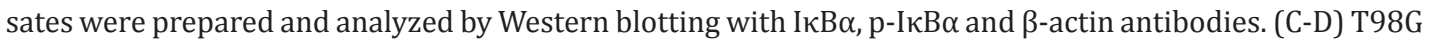
cells were wounded and exposed to IR ( 5 Gy) or left untreated along with incubation with Bay-11 for $4 \mathrm{~h}$ as indicated. The migrated distance was determined at $0 \mathrm{~h}$ and $20 \mathrm{~h}$ and quantitation as shown in B. (E) T98G cells were treated as in (C). MTT assays were performed at 12, 24 and 48 h post-treatment. (F) T98G cells were treated as in (C). At $20 \mathrm{~h}$ post-treatment, the cells were harvested and stained with FITC-Annexin V and PI followed by analysis with flow cytometry. The percentage of Annexin $\mathrm{V}^{+} / \mathrm{PI}^{-}$cells and Annexin $\mathrm{V}^{+} / \mathrm{PI}^{+}$ cells from three independent experiments was shown. ${ }^{*}, p<0.05$; ${ }^{* *}, p<0.01$; ns: no significant difference.

damage significantly enhanced the activation of NF- $\mathrm{KB}$ reporter in both T98G and U87MG cells (Fig.1B). Since DNA damage-dependent NF- $\kappa B$ activation is associated with the phosphorylation of IкB $\alpha$ and its subsequent ubiquitin-dependent degradation [14], we examined IкB $\alpha$ phosphorylation and degradation at different time points post-IR. We found I $\mathrm{B} \alpha$ phosphorylation was activated in T98G and U87MG cells by IR. Consistently, the levels of IKB $\alpha$ were reduced upon IR exposure (Fig. 1C). Given that TAK1 is upstream of IKK and

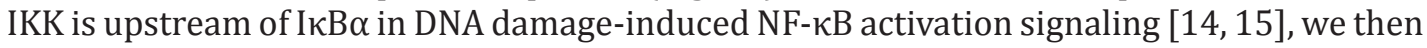
analyzed IKK phosphorylation and TAK1 phosphorylation after IR exposure in both T98G and U87MG cells. As expected, both IKK phosphorylation and TAK1 phosphorylation were activated upon IR exposure (Fig. 1D). Taken together, this evidence suggested IR treatment can effectively induce NF- $\mathrm{BB}$ activation in both T98G and U87MG cells.

Inhibition of NF- $\kappa B$ activity increases radiation sensitivity of T98G cells

As mentioned above, IR exposure can induce NF- $\kappa B$ activation in human GBM cells.

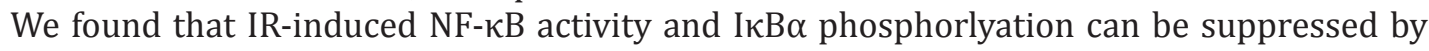
IKK/IKB $\alpha$ inhibitor (Bay-11) (Fig. 2A and B), suggesting that IR treatment can induce NF$\kappa B$ activation in a manner dependent on the activation of IKK. NF- $\kappa B$ activation has been 


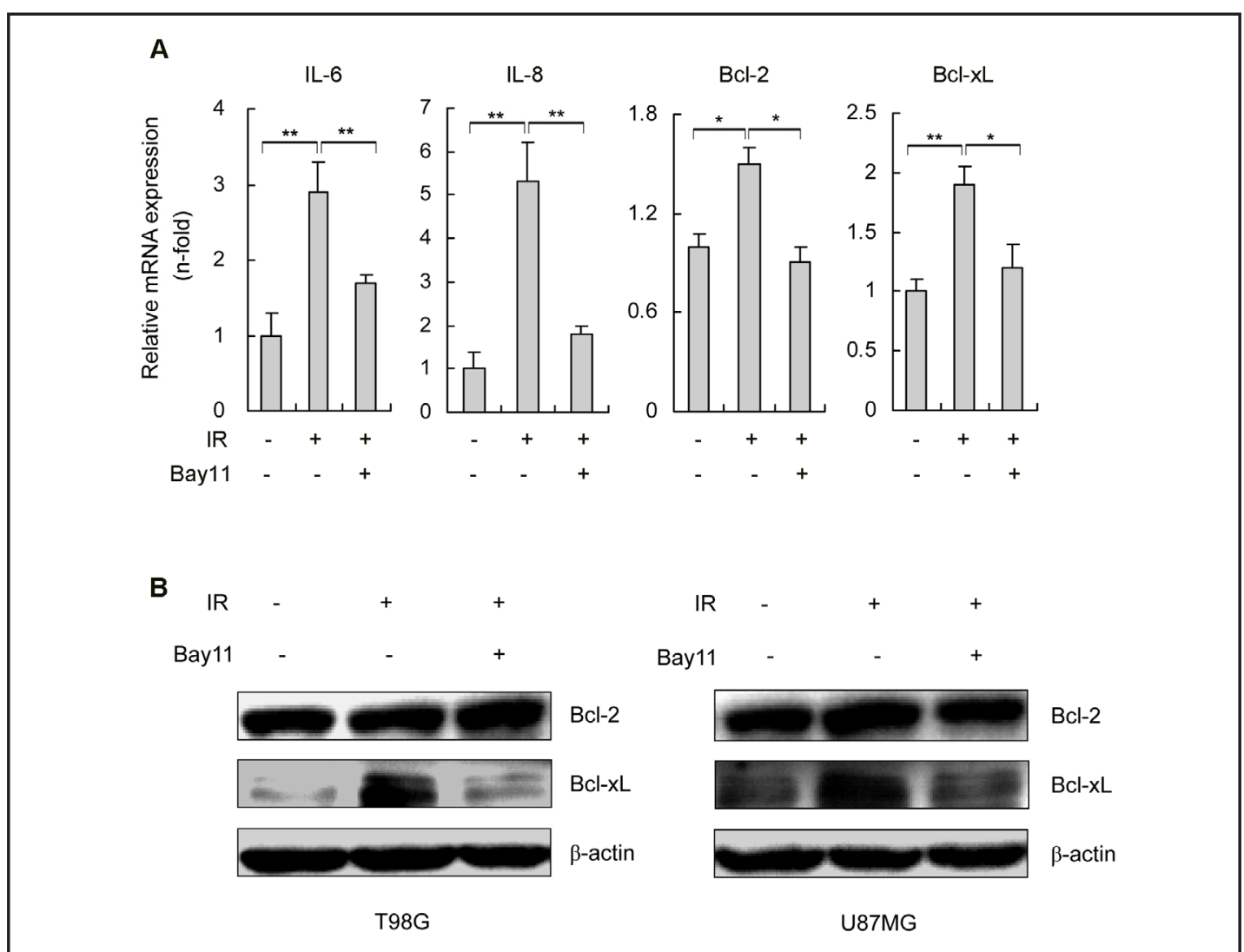

Fig. 3. IR-induced NF- $\kappa B$-dependent gene expression in T98G and U87MG cells. (A) T98G cells were treated with IR (5 Gy) or left untreated along with incubation with Bay-11 for $4 \mathrm{~h}$ as indicated. The expressions of IL-6, IL-8, Bcl-2 and Bcl-xL were evaluated by qRT-PCR with GAPDH as an endogenous control. *,$p<0.05$; $* *, p<0.01$. (B) T98G or U87MG cells were treated as in (A). At 24 h post-treatment, cell lysates were prepared and the protein levels of Bcl-2 and Bcl-xL were analyzed by Western blotting with specific antibodies as indicated.

associated with therapeutic resistance in multiple human malignancies [6]. Therefore, we examined the effects of IR-induced NF- $\kappa B$ on cell proliferation and apoptotic death in T98G cells. As shown in Fig. 2C, D, and E, IR treatment had no significant effect on invasion and proliferation of T98G cells. However, inhibition of NF- $\kappa$ B activity by Bay11 attenuated invasion $(\mathrm{p}<0.05)$ and proliferation $(\mathrm{p}<0.01)$ in T98G cells upon IR exposure. Because NF$\kappa B$ is well known to promote cell survival via inhibiting apoptosis [11], we reasoned that inhibition of NF- $\mathrm{KB}$ with Bay11 may enhance apoptosis in T98G cells. Indeed, treatment with Bay11 significantly increased apoptosis in T98G cells following IR exposure (Fig. 2F). Together, these data suggested that DNA damage-induced NF- $\kappa B$ activation may play a critical role in modulating radioresistance in human GBM cells.

IR up-regulates $N F-\kappa B$-dependent proinflammatory cytokines and anti-apoptotic genes expression in GBM cells

To further characterize the functional significance of activation of NF- $\kappa \mathrm{B}$ signaling in cancer progress upon radiotherapy, we analyzed levels of NF- $\kappa \mathrm{B}$-dependent proinflammatory cytokines IL-6 and IL-8 and anti-apoptotic genes Bcl-2 and Bcl-xL in IR-treated T98G cells by qRT-PCR. As expected, IR treatment stimulated the transcription of IL-6, IL-8, Bcl-2 and Bcl-xL; and NF- $\kappa$ B inhibitor Bay11 attenuated IR-induced upregulation of IL-6, IL-8, Bcl-2 and Bcl-xL (Fig. 3A). We also detected the protein levels of Bcl-2 and Bcl-xL in both T98G and U87MG cells. Bcl-xL, but not Bcl-2, was observed to be upregulated by IR and attenuated by 


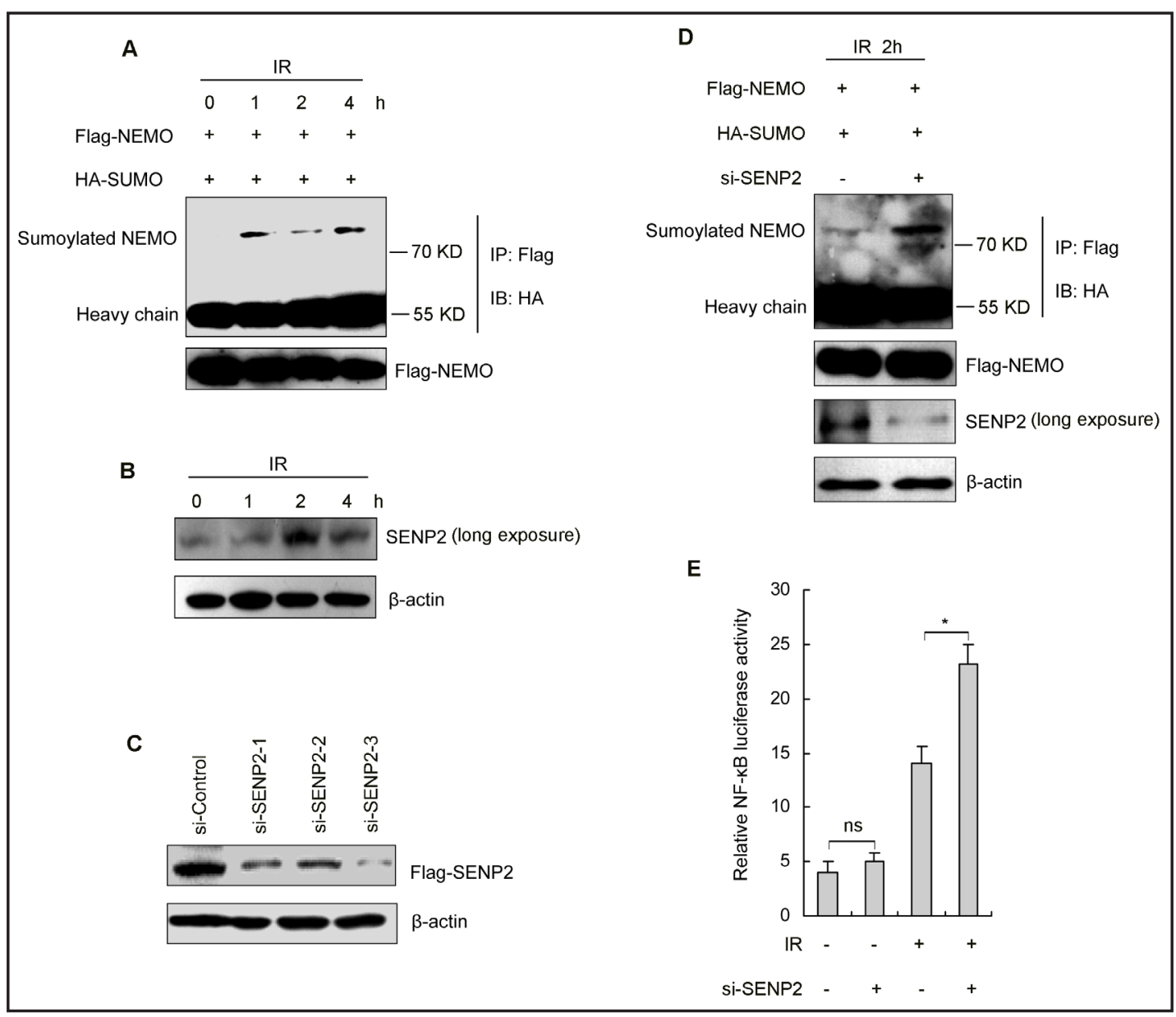

Fig. 4. Knockdown of SENP2 enhances IR-induced NEMO SUMOylation and NF- $\kappa$ B activation. (A) T98G cells were transfected with Flag-NEMO and HA-SUMO for $28 \mathrm{~h}$ followed by the treatment with IR ( 5 Gy). At 0, 1, 2 and $4 \mathrm{~h}$ post-IR, the cell lysates were extracted and IP with anti-Flag antibody followed by IB with HA or Flag antibody. (B) T98G cells were treated with IR (5 Gy). Whole-cell lysates were prepared at the indicated time points and the expressions of SENP2 were analyzed by Western blotting with anti-SENP2 antibody. (C) T98G cells were transfected with Flag-SENP2 and siRNA control or si-SENP2 (si-SENP2-1, -2 and -3) for 24 $\mathrm{h}$ followed by the re-transfection with siRNA control or si-SENP2 for another $28 \mathrm{~h}$. The levels of Flag-SENP2 were detected by Western Blot with Flag antibody. (D) T98G cells were transfected with siRNA control or si-SENP2 (a mixture of si-SENP2-1, -2 and -3) for $24 \mathrm{~h}$ followed by the co-transfection of Flag-NEMO, HA-SUMO and si-SENP2 or control siRNA for another $28 \mathrm{~h}$. The cells were treated with IR (5 Gy) for $2 \mathrm{~h}$ and the cell lysates were extracted and IP with anti-Flag antibody followed by IB with HA or Flag antibody. The expressions of SENP2 were evaluated with anti-SENP2 antibody. (E) T98G cells were transfected with control siRNA or si-SENP2 (a mixture of si-SENP2-1, -2 and -3) for $24 \mathrm{~h}$ followed by the co-transfection of pNF- $\kappa B$-Luc, pRL-TK and si-SENP2 or control siRNA for another $28 \mathrm{~h}$. The cells were then exposed to IR or left untreated. At $8 \mathrm{~h}$ post-IR, the cells were harvested, and a luciferase assay was performed. *, $p<0.05$; n.s.: no significant difference.

Bay11 treatment (Fig.3B). Together, these data indicated that DNA damage leads to enhanced production of proinflammatory cytokines including IL-6 and IL-8, and anti-apoptotic gene $\mathrm{Bcl}-\mathrm{xL}$ in GBM cells in a NF- $\kappa \mathrm{B}$ dependent manner.

Knockdown of SENP2 enhances IR-induced NEMO SUMOylation and NF- $K B$ activation Because SUMOylation of NEMO is critical for NF- $\kappa B$ activation in response to DNA damage [14], we reasoned that IR exposure would increase NEMO SUMOylation. To test this KARGER 


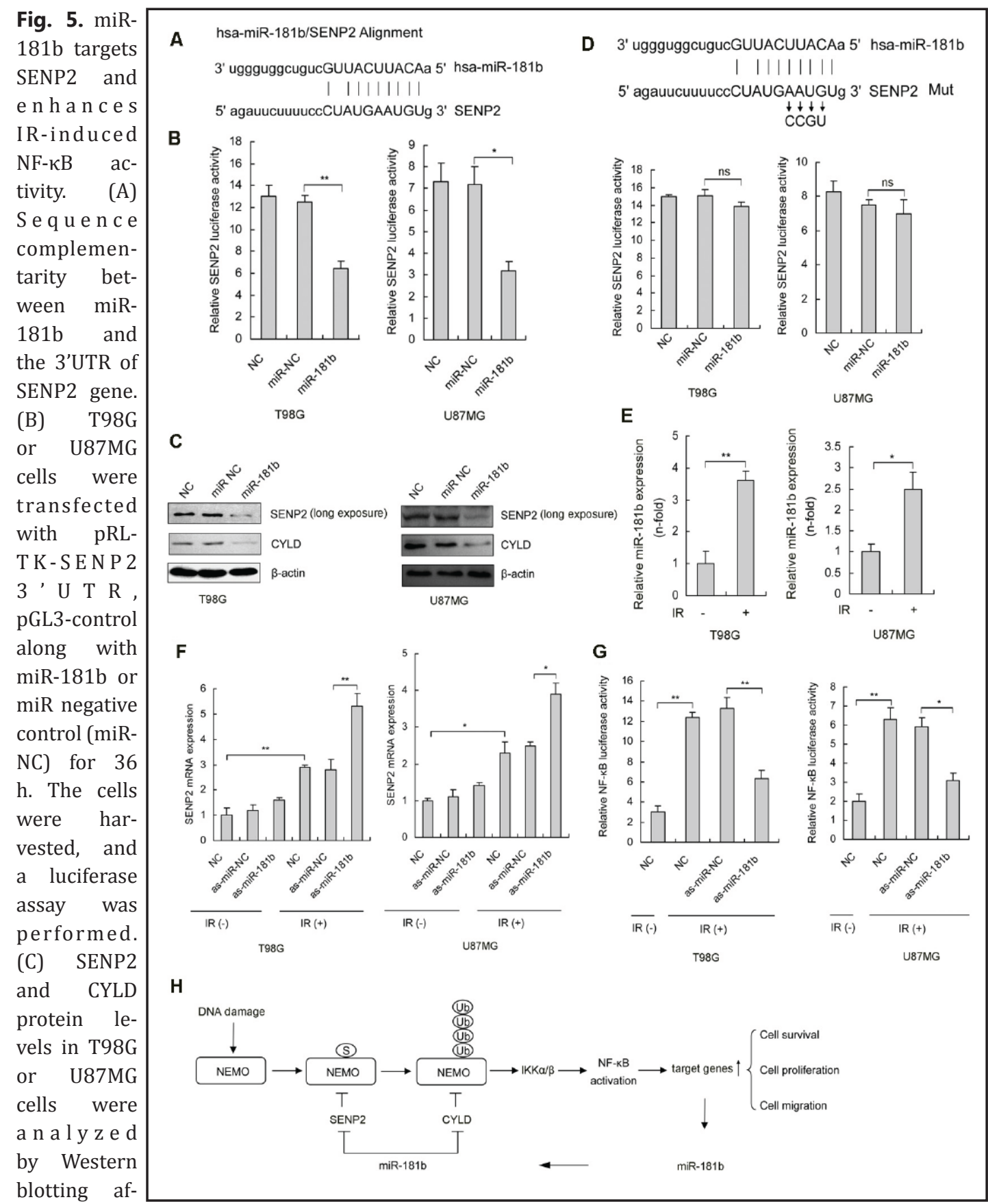

ter transfection with miR-181b or miR-NC for $48 \mathrm{~h}$. (D) T98G or U87MG cells were transfected with pRL-TK- SENP2 3'UTR mutant as indicated, pGL3-control along with miR-181b or miR-NC for $36 \mathrm{~h}$. The cells were harvested, and a luciferase assay was performed. (E) T98G or U87MG cells were treated with IR ( $5 \mathrm{~Gy}$ ) for $2 \mathrm{~h}$ or left untreated. The expressions of miR-181b were evaluated by qRT-PCR with U6 as an endogenous control. (F) T98G or U87MG cells were transfected with miR-181b inhibitor (as-miR$181 \mathrm{~b}$ ) or miR-181b inhibitor control (as-miR-NC) followed by IR treatment or left untreated. The mRNA levels of SENP2 were evaluated by qRT-PCR with GAPDH as an endogenous control. (G) T98G or U87MG cells were transfected with pNF-kB-Luc, pRL-TK along with miR-181b inhibitor (as-miR-181b) or miR$181 \mathrm{~b}$ inhibitor control (as-miR-NC) as indicated. At $36 \mathrm{~h}$ post-transfection, the cells were exposed to IR or left untreated for another $6 \mathrm{~h}$, and a luciferase assay was performed. $(\mathrm{H})$ Diagram illustrating a proposed model for IR-induced NF- $\mathrm{KB}$ activation in human GBM cells. *, $p<0.05$; **, $p<0.01$; n.s.: no significant difference. 
predication, we transfetced T98G cells with Flag-NEMO and HA-SUMO and exposed the cells to IR. At $0,1,2$ and $4 \mathrm{~h}$ post-IR, we detected NEMO SUMOylation by immunoprecipitation assay and found that NEMO SUMOylation was transiently induced at $1 \mathrm{~h}$ followed by a decrease at $2 \mathrm{~h}$. Interestingly NEMO SUMOylation was re-induced at $4 \mathrm{~h}$ after IR (Fig. 4A). SENP2 is the primary SUMO protease that deSUMOylates NEMO and an inhibitor of constitutive NF- $\kappa \mathrm{B}$ activation induced by DNA damage. We therefore wondered whether the observed alternation in NEMO SUMOylation in T98G cells upon IR exposure could be correlated with alternations of SENP2 expression. Western blots assays showed that the abundance of SENP2 was enhanced upon IR exposure at $2 \mathrm{~h}$ and reduced at $4 \mathrm{~h}$ post-IR, in contrast to NEMO SUMOylation seen in these cells (Fig. 4B). We then intended to study the effects of SENP2 knockdown on IR-induced NEMO SUMOylation. To this end, three siRNAs against SENP2 (siSENP2-1, -2 and -3) were synthesized and cotransfected respectively with Flag-SENP2 to T98G cells. As shown in Fig. 4C, all of these siRNAs decreased the expression of Flag-tagged SENP2 with si-SENP2-1 and si-SENP2-3 showing high knockdown efficiency. In the following experiments, to increase knockdown efficiency, the three siRNAs targeting SENP2 were transfected as a mixture and re-transfected after $24 \mathrm{~h}$. Our results in Fig. 4D indicated that SENP2 knockdown increased the levels of NEMO SUMOylation upon IR exposure, confirming the negative regulatory roles of SENP2 on NEMO SUMOylation. Since NEMO SUMOylation is required for DNA damage-induced NF- $\mathrm{kB}$ activation and SENP2 inhibits NEMO SUMOylation,

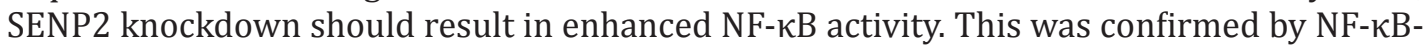
Luc reporter assay. IR exposure induced strong NF- $\kappa$ B activity in both control siRNAs and siSENP2 transfected T98G cells, and such activity was enhanced when endogenous SENP2 was knocked down (Fig. 4E). Taken together, these results suggested SENP2 inhibited IR-induced $\mathrm{NF}-\kappa \mathrm{B}$ activation in human GBM cells by deSUMOylation of NEMO.

\section{miR-181b increases IR-induced NF- $\kappa B$ activity by targeting SENP2}

The results above indicate that SENP2 levels correlate with NF- $\kappa B$ activity in IR-treated cells. As the observation that SENP2 expression was induced upon IR exposure at $2 \mathrm{~h}$ and reduced at $4 \mathrm{~h}$ post-IR, we wonder whether the levels of SENP2 could be regulated by an inducible microRNA. To test this possibility, we first performed bioinformatics analysis to predict the microRNAs targeting SENP2. miR-181b was selected for the further study because it has been shown to target cylindromatosis (CYLD) that functions as a deubiquitinating enzyme to inhibit TAK1 and IKK activation in NF- $\kappa B$ signaling pathway [23]. Sequence complementarity between miR-181b and SENP2 gene 3'UTR was shown in Fig. 5A. We then confirmed whether miR-181b targeted SENP2. To this end, we cloned SENP2 3'UTR into the pRL-TK vector immediately downstream of the firefly luciferase gene to construct the SENP2-luc reporter plasmid. T98G or U87MG cells were transfected with SENP2-luc and pGL-3-control which used as an internal control, along with miR-181b or its negative control. miR-181b overexpression significantly decreased the luciferase activity of SENP-luc vector when compared with miR control in both T98G and U87MG cells (Fig. 5B). We further investigated whether miR-181b regulated the protein levels of SENP2. T98G or U87MG cells were transfected with miR-181b or its negative control. The expression of SENP2 and the validated miR-181b target gene CYLD were detected with Western blotting using specific antibodies. We found both of the protein levels of SENP2 and CYLD were decreased by over expression of miR-181b in both T98G and U87MG cells (Fig. 5C). To further confirm the direct targeting of SENP2 by miR-181b, we constructed SENP2-mut-luc vector that carrying the mutant SENP2 3'UTR. Dual-luciferase assay indicated that miR-181b can not suppress the luciferase activity of SENP-mut-luc when compared with miR control (Fig. 5D). These results indicated that SENP2 was a direct target of miR-181b. To confirm the expression of miR-181b in GBM cells, we analyzed levels of miR-181b in T98G and U87MG cells with or without IR exposure by qRT-PCR through measuring mature miR-181b. As shown in Fig.5E, IR treatment significantly increased miR-181b expression, suggesting that miR-181b can be induced by DNA damage in human GBM cells. We then investigated the effects of miR-181b on

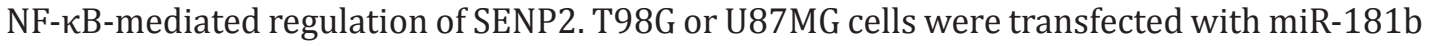


inhibitor (as-miR-181b) or its negative control (as-miR-NC) followed by IR treatment or left untreated. The mRNA levels of SENP2 were then detected by qRT-PCR. As shown in Fig.5F, IR triggers the expression of SENP2, and suppression of miR-181b expression resulted in upregulation of IR-induced SENP2 expression, suggesting that miR-181b regulated directly SENP2 expression during IR exposure. To further investigate the functional consequence of enhanced expression of miR-181b in GBM cells upon IR exposure, we transfected T98G or U87MG cells with miR-181b inhibitor and performed NF- $\kappa B$-Luc reporter assay. As shown in Fig. 5G, suppression of miR-181b expression inhibited IR-induced NF- $\kappa B$ activity. Therefore, miR-181b may function as a positive regulator on the feedback loop of NF- $\kappa B$ activation via targeting SENP2 in GBM cells when exposed to DNA damaging agents.

\section{Discussion}

Genotoxic treatment-induced NF- $\kappa \mathrm{B}$ activation has been associated with acquired therapeutic resistance in some types of cancer $[6,28,29]$. Here, we provide evidence that IR-induced DNA damage enhanced NF- $\kappa B$ activity in human GBM cells. Increased NF$\kappa \mathrm{B}$ activation up-regulates anti-apoptotic genes and proinflammatory genes which may contribute to cancer progress. Furthermore, the oncogenic miR-181b is also induced by IR which functions as a positive regulator on NF- $\mathrm{KB}$ activation by targeting SENP2.

Our model for sustained NF- $\mathrm{KB}$ activity and how NF- $\mathrm{\kappa B}$ contributes to acquired therapeutic resistance in human GBM cells upon IR exposure was present in Fig. 5H. Upon DNA damage, nuclear events trigger post-translational modifications of NEMO such as SUMOylation and ubiquitination. These modifications then mediate the activation of TAK1 and IKK $\alpha / \beta$ to promote NF- $\kappa B$ signaling [14]. Activated NF- $\kappa B$ up-regulates a cohort of genes involved in cell survival, proliferation and migration. Moreover, the increased NF- $\kappa B$ activation also promotes miR-181b transcription. miR-181b induction in turn enhances NF$\kappa \mathrm{B}$ activity through directly targeting SUMO protease SENP2 and deubiquitinating enzyme CYLD, thereby forming a positive feedback loop to drive NF- $\kappa B$ activation during IR exposure.

NF- $\kappa B$ has a dual function during tumorgenesis. In the early stages, the tightly regulated $\mathrm{NF}-\kappa \mathrm{B}$ induces cellular apoptosis in response to oncogene activation or cellular stress and hence functions as a tumor suppressor [6]. However, during tumor progression high levels of inflammatory cytokines in the microenvironment of a solid tumor or accumulated defects in the pathways regulating NF- $\kappa B$ will contribute to aberrant NF- $\kappa B$ activity [30, 31]. Aberrant activation of NF- $\mathrm{KB}$ promotes tumorigenesis through its ability to induce the expression of antiapoptotic genes such as Bcl-xL and Bcl-2, the cyclin protein Cyclin D1, cellular adhesion molecules such as ICAM-1 and VCAM-1 and chemokine receptors such as CXCR4 to promote tumor cell survival, proliferation, angiogenesis, invasion and metastasis, and hence functions as a tumor promoter [11, 32, 33]. In particular, aberrant NF- $\mathrm{\kappa B}$ activation may confer resistance to chemotherapy drugs and ionizing radiation because NF- $\kappa B$ inhibits apoptosis which induced by these treatment to kill cancer cells. Thus, inhibition of NF- $\kappa B$ could represent a potentially exciting new therapy of cancer.

MicroRNAs play critical regulatory roles in oncogenesis. The development of most human cancers was observed to be associated with dysregulated miRNAs expression. miR$181 \mathrm{~b}$ has been shown to be down regulated in GBM. However, lower levels of miR-181b in glioblastoma were positively correlated with response to radiotherapy /temozolomide (TMZ) treatment in glioblastoma patients [34]. These observations suggested that the function of miR-181b may be unique, depending on the type of tumor and cellular context. A study presented by Iliopoulos et al indicated that STAT3, a transcription factor activated by IL-6, directly activated miR-21 and miR-181b. miR-21 and miR-181b inhibited PTEN and CYLD respectively, leading to increased NF- $\kappa B$ activity to switch inflammation to cancer [23]. Here, we observed that IR exposure can induce miR-181b expression. Based on the result that IR-induced NF- $\mathrm{KB}$ promoted IL-6 expression in GBM cells, we hypothesized that IL-6/ Stat3 pathway may play essential roles in up-regulation of miR-181b in GBM cells upon IR exposure. The detailed mechanism remained for further study.

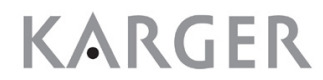


Xu et al.: Radiation Induced NF-kB Functions as a Tumor Promoter

Together, our data indicate that genotoxic stress-induced NF- $\kappa \mathrm{B}$ activation and consequent miR-181b up-regulation play a pivotal role in modulating radiation responsiveness of glioblastoma. Interfering with genotoxic NF- $\kappa \mathrm{B}$ signaling may serve as a promising strategy to antagonize glioblastoma therapeutic resistance.

\section{Acknowledgments}

This project was supported by the National Natural Science Foundation of China (81172180).

\section{Disclosure Statement}

The authors declare that they have no conflict of interest.

\section{References}

1 Sagiyama K, Mashimo T, Togao O, Vemireddy V, Hatanpaa KJ, Maher EA, Mickey BE, Pan E, Sherry AD, Bachoo RM, Takahashi M: In vivo chemical exchange saturation transfer imaging allows early detection of a therapeutic response in glioblastoma. Proc Natl Acad Sci U S A 2014;111:4542-4547.

-2 Stupp R, Mason WP, van den Bent MJ, Weller M, Fisher B, Taphoorn MJ, Belanger K, Brandes AA, Marosi C, Bogdahn U, Curschmann J, Janzer RC, Ludwin SK, Gorlia T, Allgeier A, Lacombe D, Cairncross JG, Eisenhauer E, Mirimanoff RO: Radiotherapy plus concomitant and adjuvant temozolomide for glioblastoma. N Engl J Med 2005;352:987-996.

-3 Gray GK, McFarland BC, Nozell SE, Benveniste EN: Nf-kappab and stat3 in glioblastoma: Therapeutic targets coming of age. Expert Rev Neurother 2014;14:1293-1306.

-4 Ramirez YP, Weatherbee JL, Wheelhouse RT, Ross AH: Glioblastoma multiforme therapy and mechanisms of resistance. Pharmaceuticals (Basel) 2013;6:1475-1506.

5 Barnes PJ, Karin M: Nuclear factor-kappab: A pivotal transcription factor in chronic inflammatory diseases. N Engl J Med 1997;336:1066-1071.

6 Perkins ND: Nf-kappab: Tumor promoter or suppressor? Trends Cell Biol 2004;14:64-69.

7 Yang L, Zhang J, Yan C, Zhou J, Lin R, Lin Q, Wang W, Zhang K, Yang G, Bian X, Zeng A: Sirt1 regulates cd40 expression induced by tnf-alpha via nf-kb pathway in endothelial cells. Cell Physiol Biochem 2012;30:1287-1298.

8 Wang H, Cho CH: Effect of nf-kappab signaling on apoptosis in chronic inflammation-associated carcinogenesis. Curr Cancer Drug Targets 2010;10:593-599.

-9 Baldwin AS: Regulation of cell death and autophagy by ikk and nf-kappab: Critical mechanisms in immune function and cancer. Immunol Rev 2012;246:327-345.

10 Kim JY, Lee S, Hwangbo B, Lee CT, Kim YW, Han SK, Shim YS, Yoo CG: Nf-kappab activation is related to the resistance of lung cancer cells to tnf-alpha-induced apoptosis. Biochem Biophys Res Commun 2000;273:140-146.

-11 Xiao G, Fu J: Nf-kappab and cancer: A paradigm of yin-yang. Am J Cancer Res 2011;1:192-221.

12 Sakuma Y, Yamazaki Y, Nakamura Y, Yoshihara M, Matsukuma S, Koizume S, Miyagi Y: Nf-kappab signaling is activated and confers resistance to apoptosis in three-dimensionally cultured egfr-mutant lung adenocarcinoma cells. Biochem Biophys Res Commun 2012;423:667-671.

13 Barkett M, Gilmore TD: Control of apoptosis by rel/nf-kappab transcription factors. Oncogene 1999;18:6910-6924.

14 McCool KW, Miyamoto S: DNA damage-dependent nf-kappab activation: Nemo turns nuclear signaling inside out. Immunol Rev 2012;246:311-326.

15 Hinz M, Stilmann M, Arslan SC, Khanna KK, Dittmar G, Scheidereit C: A cytoplasmic atm-traf6-ciap1 module links nuclear DNA damage signaling to ubiquitin-mediated nf-kappab activation. Mol Cell 2010;40:63-74. 
Xu et al.: Radiation Induced NF-KB Functions as a Tumor Promoter

16 Wu ZH, Wong ET, Shi Y, Niu J, Chen Z, Miyamoto S, Tergaonkar V: Atm- and nemo-dependent elks ubiquitination coordinates tak1-mediated ikk activation in response to genotoxic stress. Mol Cell 2010;40:75-86.

17 Lee MH, Mabb AM, Gill GB, Yeh ET, Miyamoto S: Nf-kappab induction of the sumo protease senp2: A negative feedback loop to attenuate cell survival response to genotoxic stress. Mol Cell 2011;43:180-191.

18 Morris JR, Boutell C, Keppler M, Densham R, Weekes D, Alamshah A, Butler L, Galanty Y, Pangon L, Kiuchi T, Ng T, Solomon E: The sumo modification pathway is involved in the brca1 response to genotoxic stress. Nature 2009;462:886-890.

19 Taganov KD, Boldin MP, Chang KJ, Baltimore D: Nf-kappab-dependent induction of microrna mir146, an inhibitor targeted to signaling proteins of innate immune responses. Proc Natl Acad Sci U S A 2006;103:12481-12486.

20 Niu J, Shi Y, Tan G, Yang CH, Fan M, Pfeffer LM, Wu ZH: DNA damage induces nf-kappab-dependent microrna-21 up-regulation and promotes breast cancer cell invasion. J Biol Chem 2012;287:21783-21795.

-21 Fu J, Xu X, Kang L, Zhou L, Wang S, Lu J, Cheng L, Fan Z, Yuan B, Tian P, Zheng X, Yu C, Ye Q, Lv Z: Mir30a suppresses breast cancer cell proliferation and migration by targeting eya2. Biochem Biophys Res Commun 2014;445:314-319.

22 Ma X, Becker Buscaglia LE, Barker JR, Li Y: Micrornas in nf-kappab signaling. J Mol Cell Biol 2011;3:159166.

23 Iliopoulos D, Jaeger SA, Hirsch HA, Bulyk ML, Struhl K: Stat3 activation of mir-21 and mir-181b-1 via pten and cyld are part of the epigenetic switch linking inflammation to cancer. Mol Cell 2010;39:493-506.

-24 Gao S, Song L, Li J, Zhang Z, Peng H, Jiang W, Wang Q Kang T, Chen S, Huang W: Influenza a virusencoded ns1 virulence factor protein inhibits innate immune response by targeting ikk. Cell Microbiol 2012;14:1849-1866.

-25 Hildeman DA, Zhu Y, Mitchell TC, Bouillet P, Strasser A, Kappler J, Marrack P: Activated t cell death in vivo mediated by proapoptotic bcl-2 family member bim. Immunity 2002;16:759-767.

26 Livak KJ, Schmittgen TD: Analysis of relative gene expression data using real-time quantitative pcr and the 2(-delta delta c(t)) method. Methods 2001;25:402-408.

27 Mailand N, Bekker-Jensen S, Faustrup H, Melander F, Bartek J, Lukas C, Lukas J: Rnf8 ubiquitylates histones at DNA double-strand breaks and promotes assembly of repair proteins. Cell 2007;131:887-900.

-28 Bhat KP, Balasubramaniyan V, Vaillant B, Ezhilarasan R, Hummelink K, Hollingsworth F, Wani K, Heathcock L, James JD, Goodman LD, Conroy S, Long L, Lelic N, Wang S, Gumin J, Raj D, Kodama Y, Raghunathan A, Olar A, Joshi K, Pelloski CE, Heimberger A, Kim SH, Cahill DP, Rao G, Den Dunnen WF, Boddeke HW, Phillips HS, Nakano I, Lang FF, Colman H, Sulman EP, Aldape K: Mesenchymal differentiation mediated by nf-kappab promotes radiation resistance in glioblastoma. Cancer cell 2013;24:331-346.

29 Tavora B, Reynolds LE, Batista S, Demircioglu F, Fernandez I, Lechertier T, Lees DM, Wong PP, Alexopoulou A, Elia G, Clear A, Ledoux A, Hunter J, Perkins N, Gribben JG, Hodivala-Dilke KM: Endothelial-cell fak targeting sensitizes tumours to DNA-damaging therapy. Nature 2014;514:112-116.

-30 Karin M, Cao Y, Greten FR, Li ZW: Nf-kappab in cancer: From innocent bystander to major culprit. Nat Rev Cancer 2002;2:301-310.

-31 Wilkinson KD: Signal transduction: Aspirin, ubiquitin and cancer. Nature 2003;424:738-739.

-32 Wang D, Liu D, Gao J, Liu M, Liu S, Jiang M, Liu Y, Zheng D: Trail-induced mir-146a expression suppresses cxcr4-mediated human breast cancer migration. Febs J 2013;280:3340-3353.

-33 Hou CH, Lin FL, Tong KB, Hou SM, Liu JF: Transforming growth factor alpha promotes osteosarcoma metastasis by icam-1 and pi3k/akt signaling pathway. Biochem Pharmacol 2014;89:453-463.

34 Slaby O, Lakomy R, Fadrus P, Hrstka R, Kren L, Lzicarova E, Smrcka M, Svoboda M, Dolezalova H, Novakova J, Valik D, Vyzula R, Michalek J: Microrna-181 family predicts response to concomitant chemoradiotherapy with temozolomide in glioblastoma patients. Neoplasma 2010;57:264-269. 